\title{
History of globulettes in the Milky Way
}

\author{
Tiia Grenman $^{1}$ [D $\cdot$ Erik Elfgren $^{1} \cdot$ Hans Weber ${ }^{1}$
}

Received: 14 August 2017 / Accepted: 7 December 2017 / Published online: 10 January 2018

(c) The Author(s) 2018. This article is published with open access at Springerlink.com

\begin{abstract}
Globulettes are small (radii $<10 \mathrm{kAU}$ ) dark dust clouds, seen against the background of bright nebulae. A majority of the objects have planetary mass. These objects may be a source of brown dwarfs and free floating planetary mass objects in the galaxy. In this paper we investigate how many globulettes could have formed in the Milky Way and how they could contribute to the total population of free floating planets. In order to do that we examine H-alpha images of $27 \mathrm{H} \mathrm{II}$ regions. In these images, we find 778 globulettes.

We find that a conservative value of the number of globulettes formed is $5.7 \times 10^{10}$. If $10 \%$ of the globulettes form free floating planets then they have contributed with $5.7 \times 10^{9}$ free floating planets in the Milky Way. A less conservative number of globulettes would mean that the globulettes could contribute $2.0 \times 10^{10}$ free floating planets. Thus the globulettes could represent a non-negligible source of free floating planets in the Milky Way.
\end{abstract}

Keywords Free Floating Planets · Globulettes · H II regions · ISM · Milky Way

\section{Introduction}

$\mathrm{H}$ II regions, surrounding young stellar clusters are driven by massive $\mathrm{O}$ and early $\mathrm{B}$ stars. These regions are associated with dusty gas formations, such as pillars, 'elephanttrunks' and isolated dark clouds seen in different sizes and shapes, from large irregular blocks and fragments to smaller more roundish objects. These small-sized globules within

T. Grenman

Tiia.Grenman@1tu.se

1 Luleå University of Technology, Luleå, SE-971 87 Luleå, Sweden
H II regions were first observed by Bok and Reilly (1947), followed by Thackeray (1950) and Herbig (1974). They are dense, cold and neutral clouds with dark appearance in optical images. The pillars of gas and dust are pointing towards the ionizing sources and are usually connected to the molecular shell.

In such $\mathrm{H}$ II regions, a new distinct class of objects, globulettes, was noted by Gahm et al. (2007) and Grenman (2006). The globulettes are seen as silhouettes against the background of bright nebulae in optical images. Typically, they are roundish objects that are much smaller $(<10 \mathrm{kAU})$ than normal globules with a mass of $\lesssim 0.1 M_{\odot}$ (Gahm et al. 2007). The globulettes can also have tails, bright rims and halos. Many globulettes are quite isolated, located far from the molecular shells and dust pillars associated with the regions. However, some of the objects are connected by thin filaments to large molecular blocks or even each other. This suggests that they can either form separately, or via fragmentation of large structures. The calculated lifetime of globulettes suggests that they may survive in this harsh environment for a long time, $>10^{6} \mathrm{yrs}$ (Gahm et al. 2007; Haworth et al. 2015).

In the Carina Nebula (NGC 3372), a giant H II region, the globulettes are smaller, more dense and less massive (Grenman and Gahm 2014) than those recognized in Gahm et al. (2007) and De Marco et al. (2006). This group of globulettes may represent a more advanced evolutionary state in globulette evolution. Schneider et al. (2016) suggested that the pillars may evolve first into a globule and then into a globulette when the lower density gas has photoevaporated and left behind a dense and cold core. However, it is also speculated that globulettes might collapse in situ to form free floating planets (FFPs with $<13 M_{J}$, where $M_{J}=$ Jupiter mass) or brown dwarfs $\left(13-75 M_{J}\right)$, triggered by external forces from gas and turbulent pressure in the sur- 
rounding warm plasma and radiation pressure from stellar photons. In fact, the result by Gahm et al. (2013) from nearinfrared imaging of the Rosette Nebula, revealed very dense cores in some of the largest globulettes that might collapse to form FFPs. They also pointed out that these objects accelerate outwards from the nebula and eventually ends up in the interstellar environment.

A more recent example, where globulettes may have formed planetary mass objects was found in the Orion Nebula (M42/43, Fang et al. 2016). This nebula stands out by having both dark silhouette disks and bright compact knots. They are known as proplyds (O'Dell et al. 1993; O’Dell and Wong 1996; Bally et al. 2000).

The first reports of populations of substellar objects in interstellar space came in 1995 (Rebolo et al. 1995; Nakajima et al. 1995). Since then, a number of Jupiter-mass FFPs and FFP candidates have been found in young clusters and star formation regions (see Lucas and Roche 2000; Bihain et al. 2009; Luhman 2012; Liu et al. 2013; Peña Ramírez et al. 2016, 2012; Clanton and Gaudi 2017, and references therein). Planetary mass objects have also been found in the galactic field (Cushing et al. 2014). Since these substellar objects are both brighter and warmer at young ages (less than $10 \mathrm{Myr}$, Chabrier et al. 2000), these objects with masses of a few Jupiter masses can be seen e.g. in deep optical and near-infrared photometric surveys (Zapatero Osorio et al. 2000, 2017; Quanz et al. 2010).

Another method to find objects below the deuteriumburning-mass-limit (Jupiter-sized) that are not bound to a host star is through gravitational microlensing. This type of study has been done by the Microlensing Observation in Astrophysics group (MOA-2; Sumi et al. 2003, 2011; Freeman et al. 2015) and the Optical Gravitational Lensing Experiment (OGLE-III; Wyrzykowski et al. 2015).

The origin of these unbound objects is unclear. They may have formed in situ from a direct collapse of a molecular cloud by fragmentation, similar to star formation (e.g. Silk 1977; Bowler et al. 2011). In fact, Fang et al. (2016), Luhman et al. (2005), Boucher et al. (2016), Joergens et al. (2015), Bayo et al. (2017) have found four FFPs with accretion disk. Furthermore, planetary-mass binaries, which would be almost impossible to from through planetary formation mechanisms, have also be found by e.g. Jayawardhana and Ivanov (2006), Best et al. (2017).

FFPs may also have been formed from stellar embryos that are fragmented and photoevaporated from nearby stars (see e.g. Kroupa and Bouvier 2003; Padoan and Nordlund 2004; Whitworth and Zinnecker 2004). They may also have been ejected after they were formed in circumstellar protoplanetary disks through gravitational instabilities (e.g. Boss 2009; Stamatellos and Whitworth 2009; Li et al. 2015). Such instabilities can be caused by a triplet star system (Reipurth and Mikkola 2015) or by passage through the center of a dense star cluster (Wang et al. 2015).
In this work, we present results based on available images of H II-regions from the Hubble Space Telescope (HST) archive and taken with the Nordic Optic Telescope (NOT). We create a model of how many globulettes may have formed in the Milky Way over time and how many of these that contribute to the total FFPs population. We have organized the paper as follows. In Sect. 2, we present the result of both old and new H II regions investigated in the context of globulettes. We also present mean radii of histograms of globulettes for those $\mathrm{H} \mathrm{II}$ regions, which contain many globulettes. In Sect. 3, the parameters that are used in our model are presented. The results and discussion regarding FFPs can be found in Sect. 4 and we conclude the paper in Sect. 5 .

\section{Archival data and measurements}

In this work, we used archival H-alpha images of $16 \mathrm{H} \mathrm{II}$ regions included in earlier studies by De Marco et al. (2006), Gahm et al. (2007) and Grenman and Gahm (2014). The observations in these studies are from the HST archive and the NOT. Most images were taken with the HST instruments such as ACS/WFC, WFPC2, WFPC3/UVIS, while the NOT images were taken with the ALFOSC instrument. A brief description of these instruments is given below.

1. The Wide Field and Planetary Camera 2 (WFPC2) field of view is covered by four cameras, each of which span $800 \times 800$ pixels in size. Three of them are arranged in an L-shaped field and operated at a pixel scale of $0.1^{\prime \prime}$. The fourth one is referred to as the Planetary Camera (PC) and operates at a pixel scale of $0.046^{\prime \prime}$.

2. The Wide Field Camera 3 (WFC3) replaced the WPFC2 camera in 2009. The Ultraviolet-Visible channel (UVIS) use a mosaics of two CCDs, with $0.04 \mathrm{arcsec} / \mathrm{pixel}$, covering a $162^{\prime \prime} \times 162^{\prime \prime}$ field of view. The 2 CCDs are butted together but have a $\sim 1.4^{\prime \prime}$ gap between the two chips.

3. The Advanced Camera for Surveys (ACS/WFC) camera contains two CCDs of $2048 \times 4096$ pixels glued together with a small gap of approximately 50 pixels in between. The pixel size corresponds to $0.05^{\prime \prime}$ per pixel and the field of view is $202^{\prime \prime} \times 202^{\prime \prime}$.

4. The Andalucia Faint Object Spectrograph and Camera (ALFOSC) has a field of view of $6.5^{\prime}$, and the effective scale of the CCD detector is $0.188^{\prime \prime}$ per pixel.

The respective areas for the instruments WFPC2, WFC3, ACS and ALFOSC are estimated to 5, 7, 11 and $33 \operatorname{arcmin}^{2}$. More information about each of these instruments can be found in the manuals on the HST Web site ${ }^{1}$ and in the NOT manual. ${ }^{2}$ All images in the current study are analyzed using

\footnotetext{
${ }^{1}$ http://www.stsci.edu/hst/HST_overview/instruments.

${ }^{2}$ http://www.not.iac.es/instruments/alfosc.
} 
Table 1 Archival HST/HLA/NOT data used

\begin{tabular}{|c|c|c|}
\hline H II region & Instrument & ID \\
\hline Gum 29 & ACS/WFC & 13038 \\
\hline Gum 31 & WFPC2, ACS/WFC & 10475,9857 \\
\hline Gum 38b & WFC3/UVIS & 11360 \\
\hline IC 434 & WFPC2, ACS/WFC & $8874,9741,12812$ \\
\hline IC 2177 & ALFOSC & N/A, see Gahm et al. (2007) \\
\hline IC 2944 & WFPC2 & 7381 \\
\hline M 8 & WFPC2, ACS/WFC & $6227,11981,9857$ \\
\hline M 16 & WFPC2, ACS/WFC & $10393,13926,9091,5773$ \\
\hline M 17 & WFPC2, ACS/WFC & 6574,8992 \\
\hline M 20 & WFPC2 & 9104,11121 \\
\hline M 42/43 & WFPC2, ACS/WFC & $5469,9825,10246$ \\
\hline NGC 281 & WFPC2, ACS/WFC & $8713,10713,9857$ \\
\hline NGC 1977 & ACS/WFC & 12250 \\
\hline NGC 2174 & WFPC2, ACS/WFC & $9091,13623,9091$ \\
\hline NGC 2467 & WFPC2, ACS/WFC & 9857 \\
\hline NGC 3372 & WFPC2, ACS/WFC & $6042,11501,13390,13391,13791,10241,10475$ \\
\hline NGC 6357 & WFPC2, ACS/WFC & 9091,9857 \\
\hline NGC 6820 & ALFOSC & N/A, see Gahm et al. (2007) \\
\hline NGC 7635 & WFPC2, ACS/WFC, WFC3/UVIS & 7515,14471 \\
\hline NGC 7822 & ALFOSC & N/A, see Gahm et al. (2007) \\
\hline S106 & WFPC2, WFC3/UVIS & 5963,12326 \\
\hline S155 & WFPC2 & 5983 \\
\hline S190 & ALFOSC & N/A, see Gahm et al. (2007) \\
\hline S199 & ALFOSC & N/A, see Gahm et al. (2007) \\
\hline S273 & WFPC2, ACS/WFC, ALFOSC & 8992,5983 \\
\hline S277 & WFPC2, ACS/WFC & $5983,8992,9424$ \\
\hline Rosette Nebula & ALFOSC & N/A, see Gahm et al. (2007) \\
\hline 30 Doradus & ACS/WFC, WFC3/UVIS & 11360,12939 \\
\hline
\end{tabular}

the F656N and F658N filters, which allow for mapping circumstellar matter and detecting the dark silhouette objects, even though the F658N filter includes more nebular emission from [N II]. The HST and NOT observations from these regions are presented in Table 1 .

We characterize the angular size of the globulettes by using the SAOImage DS9 program, where we fit an ellipse to each object. Then we estimate the radius $\bar{r}$, the mean of the semi-major and semi-minor axes, expressed in kAU. In order to get a representative size distribution, a certain number of globulettes are needed. We have set the cutoff at 17 globulettes per region, and made histograms. Figure 1 shows the distributions of average radii in kAU for these $\mathrm{H}$ II regions. The average radii distribution of the all globulettes seems to fall off approximately exponentially.
Using catalogues, such as Sharpless (1959, hereafter Sharpless = S) and Gum (1955), we have investigated 319 optically visible nebulae and found $28 \mathrm{H}$ II regions that have been imaged using an $\mathrm{H}$-alpha filter. These regions are listed in Table 2, Column 1, in alphabetical order with the coordinates (Columns 2 and 3) from the Simbad database. ${ }^{3}$ The main cluster designation is given in Column 4 , and the distances from the Sun is given in Column 5, and the references in Column 6. The calculation of the H II nebula areas in Column 7 is described in Sect. 3.2 and the calculation of the observed areas in Column 8 is described in Sect. 3.3. The next to last Column in Table 2, lists the number of globulettes found in the observed area. In the last Column the

\footnotetext{
${ }^{3}$ The SIMBAD database, operated at CDS, Strasbourg, France
} 

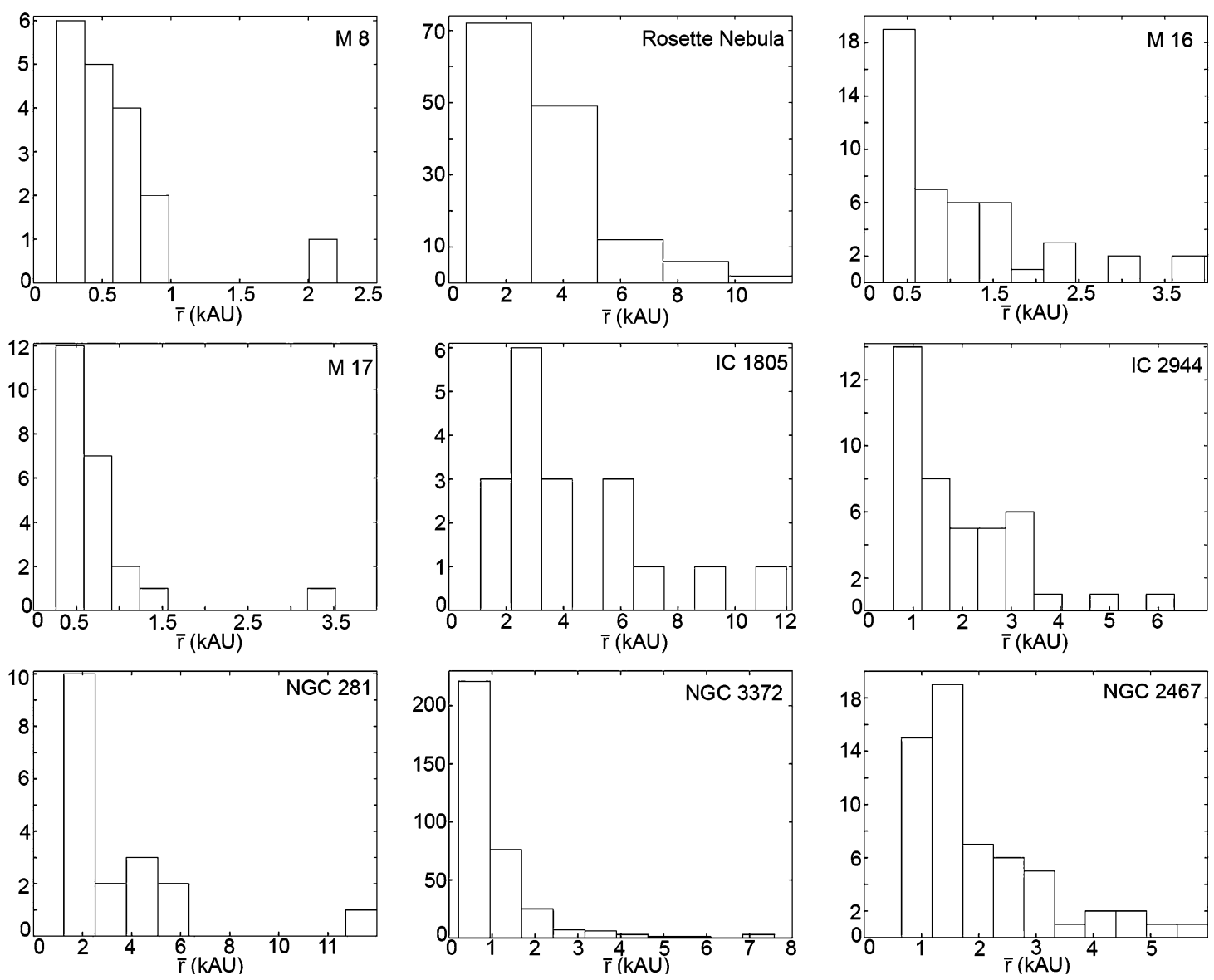

Fig. 1 The mean radii distribution for those $\mathrm{H}$ II regions containing most globulettes. The histograms peak at about $0.3 \mathrm{kAU}$

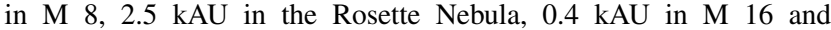

M 17, $2.5 \mathrm{kAU}$ in IC 1805, $0.9 \mathrm{kAU}$ in IC 2944, $1.9 \mathrm{kAU}$ in NGC 281, 0.6 kAU in NGC 3372 and $1.5 \mathrm{kAU}$ in NGC 2467.

\section{Model parameters}

In this section we define the different parameters needed to model the number of globulettes as shown in Table 3. To illustrate the methodology of this work we chose the Rosette Nebula as a representative example, because it contains only one main cluster, is symmetric and hold a large amount globulettes. An overview of the Rosette Complex can be found in Román-Zúñiga and Lada (2008).

\subsection{Young Universe and $\mathrm{H}$ II formation rate}

From model calculations we know that within the first relic H II regions, the massive metal free Population III (Pop III) stars evacuated the primordial gas from their minihalos (Kitayama et al. 2004; Alvarez et al. 2006; Abel et al. 2007). As the Pop III stars ended in supernova explosions, the Universe's intergalactic medium got progressively enriched with heavy metals (i.g. Greif et al. 2007; Wise and Abel 2008) and dust.

Cosmological simulations suggest that once the gas reaches the minimum or 'critical' metallicity in the range 
Table 2 List of H II regions investigated

\begin{tabular}{|c|c|c|c|c|c|c|c|c|c|}
\hline H II region & $\begin{array}{l}\text { RA } \\
(\mathrm{J} 2000.0)\end{array}$ & $\begin{array}{l}\text { Dec. } \\
(\mathrm{J} 2000.0)\end{array}$ & Main cluster & $\begin{array}{l}\text { Distance } \\
(\mathrm{kpc})\end{array}$ & Ref. & $\begin{array}{l}\text { Nebula area } \\
\operatorname{arcmin}^{2}\end{array}$ & $\begin{array}{l}\text { Obs. area } \\
\operatorname{arcmin}^{2}\end{array}$ & $\begin{array}{l}\text { Number of } \\
\text { globulettes }\end{array}$ & $\begin{array}{l}\text { Detection } \\
\text { limit }^{b}(A U)\end{array}$ \\
\hline Gum 29 & 1024 & -5746 & Westerlund 2 & 4.2 & 1 & 707 & 11 & 10 & 630 \\
\hline Gum 31 & 1037 & -5839 & NGC 3324 & 2.3 & 2 & 177 & 43 & 7 & 345 \\
\hline Gum $38 b$ & 1115 & -6115 & NGC 3603 & 6.0 & 3 & 94 & 7 & 5 & 720 \\
\hline IC 434 & 0541 & -0230 & Sigma Orionis & 0.4 & 4 & 471 & 86 & - & 60 \\
\hline IC $2177^{\mathrm{a}}$ & 0704 & -1027 & N/A & 1.3 & 5 & 314 & 33 & - & 733 \\
\hline IC 2944 & 1138 & -6322 & Collinder 249 & 2.3 & 6 & 1866 & 5 & 34 & 690 \\
\hline M 8 & 1803 & -2423 & NCC 6530,6523 & 1.3 & 7 & 1374 & 43 & 18 & 195 \\
\hline M 16 & 1818 & -1348 & NGC 6611 & 1.8 & 7 & 825 & 53 & 46 & 270 \\
\hline M 17 & 1820 & -1610 & NGC 6618 & 2.0 & 7 & 806 & 16 & 23 & 300 \\
\hline M 20 & 1802 & -2258 & NGC 6514 & 2.7 & 7 & 361 & 21 & - & 810 \\
\hline M 42/43 & 0535 & -0523 & NGC 1976, 1982 & 0.41 & 7 & 1689 & 639 & 9 & 61 \\
\hline NGC 281 & 0052 & +5634 & IC 1590 & 2.8 & 8 & 589 & 32 & 18 & 420 \\
\hline NGC 1977 & 0535 & -0449 & NGC 1973,75 & 0.41 & 9 & 314 & 44 & - & 61 \\
\hline NGC 2174 & 0609 & +2030 & NGC $2175,75 \mathrm{~s}$ & 2.2 & 12 & 589 & 35 & 15 & 330 \\
\hline NGC 2467 & 0752 & -2625 & Haffner18ab, 19 & 5.0 & 10 & 467 & 16 & 59 & 750 \\
\hline NGC 3372 & 1044 & -5953 & $\begin{array}{l}\operatorname{Tr} 14 / 15 / 16, \text { Bo10/11, } \\
\text { Cr232, Cr234/228 }\end{array}$ & 2.9 & 11 & 10179 & 953 & 343 & 435 \\
\hline NGC 6357 & 1726 & -3412 & $\begin{array}{l}\text { Pis } 24, \text { ESO 392-11, } \\
\text { ESO 393-13 }\end{array}$ & 1.7 & 7 & 911 & 17 & 11 & 255 \\
\hline NGC $6820^{\mathrm{a}}$ & 1942 & +2305 & NGC 6823 & 1.9 & 13 & 707 & 53 & 1 & 1072 \\
\hline NGC 7635 & 2320 & +6112 & [BDS2003]44 & 2.4 & 14 & 94 & 37 & 4 & 288 \\
\hline NGC $7822^{\mathrm{a}}$ & 0001 & +6725 & Berkeley 59 & 0.91 & 12 & 1884 & 72 & 9 & 513 \\
\hline S106 & 2027 & +3722 & S106 IR & 1.7 & 15 & 3 & 13 & - & 204 \\
\hline S155 & 2258 & +6231 & CepIII & 0.84 & 12 & 1178 & 10 & - & 252 \\
\hline $\mathrm{S} 190^{\mathrm{a}}$ & 0232 & +6127 & IC 1805 & 2.0 & 12 & 5281 & 189 & 18 & 1128 \\
\hline $\mathrm{S} 199^{\mathrm{a}}$ & 0251 & +6024 & IC $1848, \mathrm{Cl} 34$ & 1.8 & 12 & 5089 & 66 & - & 1015 \\
\hline $\mathrm{S} 273^{\mathrm{a}}$ & 0641 & +0953 & NGC 2264 & 0.91 & 7 & 2672 & 126 & - & 136 \\
\hline S277 & 0542 & -0155 & NGC 2024 & 0.41 & 7 & 491 & 107 & 3 & 61 \\
\hline $\begin{array}{l}\text { Rosette } \\
\text { Nebula }^{\mathrm{a}}\end{array}$ & 0631 & +0457 & NGC 2244 & 1.4 & 16 & 5281 & 570 & 145 & 790 \\
\hline $\begin{array}{l}\text { LMC: } \\
30 \text { Doradus }\end{array}$ & 0631 & +0457 & NGC 2070 & 50 & 17 & 176 & 132 & 176 & 2000 \\
\hline
\end{tabular}

References-(1) Vargas Álvarez et al. 2013; (2) Ohlendorf et al. 2013; (3) Brandner et al. 2000; (4) Mookerjea et al. 2009; (5) Bica et al. 2003; (6) Sana et al. 2011; (7) Feigelson et al. 2013, and reference therein; (8) Sato et al. 2008; (9) Menten et al. 2007; (10) Yadav et al. 2015; (11) Hur et al. 2012; (12) Foster and Brunt 2015; (13) Kharchenko et al. 2005; (14) Moore et al. 2002; (15) Schneider et al. 2007; (16) Hensberge et al. 2000; (17) Pietrzyński et al. 2013

${ }^{\mathrm{a}} \mathrm{H}$ II regions from Gahm et al. (2007)

${ }^{\mathrm{b}}$ The minimum detection size (diameter in AU) of an object, defined as 3 pixels in this article

$10^{-6}-10^{-3.5} Z_{\odot}$ which allows for more efficient cooling. An example of a low metallicity Pop II environment is the Large Magellanic Cloud (LMC), where the metallicity is $\sim 0.5 Z_{\odot}$ (Rolleston et al. 2002).

In Som et al. (2015, Fig. 11) these different metallicity values correspond to $T_{0} \sim 12 \mathrm{Gyr}$, which we set as our starting point for the calculations of the number of globulettes in the Milky Way.
In the early Universe, the formation rate of the H II regions was higher than it is now. We assume that it is proportional to the Star Formation Rate (SFR), which combined with the present day gives us the formation rate of $\mathrm{H}$ II regions in the early Universe. Marasco et al. (2015, Fig. 1), show an overview of the SFR evolution as a function of time, where the current SFR is about $\sim 5 M_{\odot} / \mathrm{yr}$. We estimate the total area under the curve, which gives us the average SFR 
Table 3 Numerical values used in the calculations

\begin{tabular}{|c|c|c|c|}
\hline Parameters/symbols & $\begin{array}{l}\text { Numerical } \\
\text { values }\end{array}$ & Description & Section \\
\hline$N_{\mathrm{H} \text { II }}$ & 27 & Number of analyzed Galactic H II regions & Table 2 \\
\hline$T_{0}$ & 12 & Starting point in time for the model (Gyrs) & 3.1 \\
\hline$f_{\mathrm{H} \mathrm{II}}$ & 2 & Correction for more $\mathrm{H}$ II regions in the early Milky Way & 3.1 \\
\hline$A_{\text {tot }}$ & 44413 & Total area of $27 \mathrm{H}$ II regions $\left(\operatorname{arcmin}^{2}\right)$ & 3.2 \\
\hline$A_{o b s}$ & 3297 & Total observed area of $27 \mathrm{H}$ II regions $\left(\operatorname{arcmin}^{2}\right)$ & 3.3 \\
\hline$f_{B}$ & 2 & Correction factor for globuletter on the backside of the nebula & 3.4 \\
\hline$T_{\mathrm{H} \text { II }}$ & 5 & Lifetime of H II regions (Myrs) & 3.5 \\
\hline$T_{\text {glob }}$ & 4 & Lifetime of a globulette (Myrs) & 3.5 \\
\hline$f_{S}$ & 2 & Correction factor between HST and NOT instruments & 3.6 \\
\hline$G_{\mathrm{NOT}}$ & 346 & Corrected number of globulettes observed with NOT & 3.6 \\
\hline$G_{\mathrm{HST}}$ & 605 & Total number of HST globulettes & Table 2 \\
\hline$n_{\mathrm{H} \mathrm{II}}$ & 10000 & Number of H II regions (present time) & 3.7 \\
\hline$G_{T o t}$ & 951 & Total corrected number of globulettes & 4 \\
\hline
\end{tabular}

between now and 10 Gyrs ago, which is twice as big as the $\mathrm{H}$ II production is today. We call this correction factor $f_{\mathrm{H} \text { II }}$, see Table 3 .

\subsection{Shapes and areas of $\mathrm{H}$ II regions}

H II regions vary in their morphology and the nebulae examined here are either roughly circular, elliptical or irregular. The angular sizes for each $\mathrm{H}$ II region were measured from large scale DDS images (POSS II/UKSTU) taken through the red filter, with a plate scale of $\sim 1^{\prime \prime}$ per pixel, available in Aladin, an interactive software Sky Atlas. ${ }^{4}$ Thereafter, the area, in $\operatorname{arcmin}^{2}$, for each H II region was estimated, see Table 2 (Column 7), where the radii were defined as the geometric mean, $\sqrt{a b}$, where $a$ and $b$ are half the apparent major and minor axes. Here, the shape of the Rosette Nebula is assumed to be circular with an area of $5281 \operatorname{arcmin}^{2}$. The total area of the $27 \mathrm{H}$ II regions was estimated to $A_{\text {tot }}=44413 \operatorname{arcmin}^{2}$.

\subsection{Observed areas of the $\mathrm{H}$ II regions}

The DDS images of the $28 \mathrm{H}$ II regions from the HST archive AstroView were overlaid with the HST apertures while the survey done with the NOT is described in Gahm et al. (2007). The observed area (Column 8 in Table 2) was calculated in $\operatorname{arcmin}^{2}$ for each nebula. For the Rosette Nebula the area was estimated to be $570 \operatorname{arcmin}^{2}$ and the total observation area of the 27 regions, $A_{o b s}$ is about $3297 \operatorname{arcmin}^{2}$.

\footnotetext{
${ }^{4}$ http://aladin.u-strasbg.fr/aladin.gml.
}

\subsection{Objects behind the $\mathrm{H} I \mathrm{I}$ regions}

We can only detect globulettes in front of the H II regions, while objects on the remote side are covered by nebular emission or hidden behind the foreground obscuring shell. Therefore, we assume that the backside of the nebula contains as many globulettes as we observe in front of the nebula. This gives a correction factor of $f_{B}=2$.

\subsection{Age estimate of the $\mathrm{H} \mathrm{II} \mathrm{regions} \mathrm{and} \mathrm{globulettes}$}

An $\mathrm{H}$ II region lasts only as long as the main sequence lifetimes of its ionizing star(s), i.e. 2-4 million years, before the radiation pressure from the hot young stars eventually drives most of the gas away and the nebula disperses. However, H II regions can live longer either in large star forming regions, involving multiple clusters, progressive star formation (e.g. the Carina Nebula, Smith et al. 2010) or when there is a spread in stellar ages, with co-existing younger ( $\sim 1$ Myr $)$ and older $(\sim 10$ Myr $)$ stars. Two examples are Gum 38b (Beccari et al. 2010) and M 16, which have cluster mean ages of about 2 Myrs but where one of the massive members is about 6 Myrs old (Hillenbrand et al. 1993). Thus by taking these conditions into account, the mean life time, $T_{\mathrm{H} \text { II }}$, for H II regions is assumed to be 5 Myrs.

The lifetimes of globulettes can be estimated to 4 Myrs, see Sect. 1.

\subsection{Spatial resolution and distance correction}

The spatial resolution of the ground based instrument NOT is lower than that of the HST. The practical globulette observation limit for the NOT telescope is about $0.8^{\prime \prime}$, whereas 
Fig. 2 Distribution of average measured radii for all globulettes found in the $\mathrm{H}$ II regions, expressed in kAU. The detection limit of $0.4 \mathrm{kAU}$ is also marked in the figure

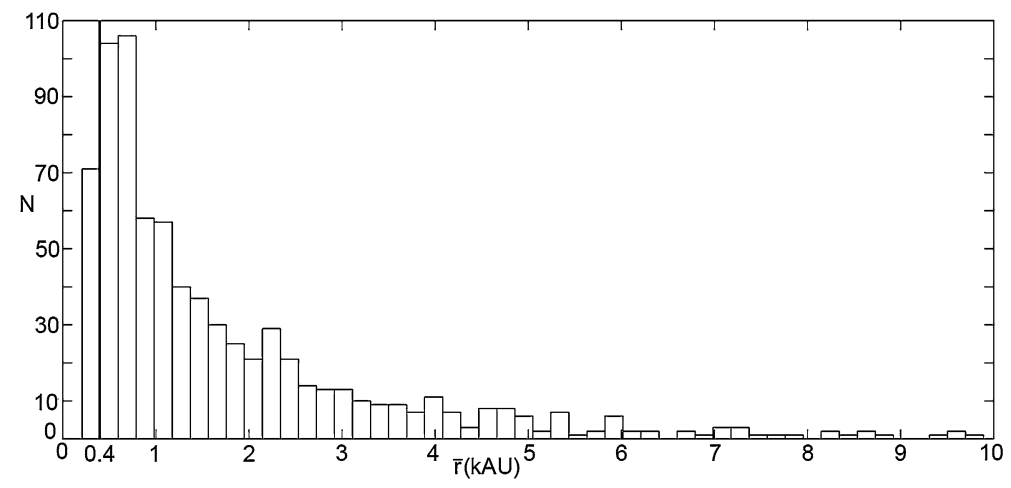

the observation limit for the HST is about $0.15^{\prime \prime}$. Thus we need to compensate for the objects that are below the resolution threshold for the NOT telescope. A histogram of the distributions of radii observed with the different instruments is shown in Fig. 2. The most significant NOT region is the Rosette nebula, which has an observation limit of $r=0.8^{\prime \prime} \times 1.4 \mathrm{kpc}=1.12 \mathrm{kAU}$. The are about as many objects above this limit in the figure as there are objects below. Therefore, the correction factor for the Rosette nebula is $f_{S} \approx 2$. For the other NOT regions, the correction factor would be only slightly different. An approximate value for the total number of globulettes within the NOT regions is $G_{\text {NOT }}=f_{S} \times 173=346$. Furthermore, some H II regions lie closer to us than others which means that we need to compensate for the missing objects at large distances. From Fig. 2, we see that the detection limit radii is $\sim 200 \mathrm{AU}$ for the more nearby regions. The only regions that are further away are NGC 2467 and Gum 38b. If we assume that doubling the distance, doubles the detection limit, the limit would be at $400 \mathrm{AU}$. In Fig. 2, we see that this correction between 200 to $400 \mathrm{AU}$ would correspond to $12 \%$ if we assume that the distribution is similar. This factor also only applies to the two regions further away. Thus, this correction factor is negligible for the current data set.

\subsection{Number of H II regions in the Milky Way}

$\mathrm{H}$ II regions evolve with time; from hypercompact (linear size $<0.01$ pc; e.g. Kurtz and Franco 2002) to ultracompact $(<0.1$ pc; e.g. Kim and Koo 2003) to compact (0.1-1 pc Wood and Churchwell 1989) and then finally to extended $\mathrm{H}$ II regions (>1 pc; Mellema et al. 2006). The evolutionary stage of a region is inferred from observations in several spectral windows. The most complete catalog today is compiled by Anderson et al. (2014, 2015), where about 8000 $\mathrm{H}$ II regions and $\mathrm{H}$ II region candidates in the Milky Way are registered. A round figure on the total number of $\mathrm{H}$ II regions can be 10000 (L.D. Andersson, personal communication, 2016).

\section{Results and discussion}

In this section, we establish a model equation for the number of globulettes that have been formed in the history of the Milky Way. We also discuss how many FFPs may form from globulettes by taking account different parameters. In addition, we also discuss briefly the role played by giant H II regions, like the Carina nebula and 30 Doradus, for producing globulettes in the early Milky Way. In our scenario, the first globulettes were formed roughly 12 Gyr ago, when there was enough metallicity to create dust, (cf. Sect. 3.1). We estimate that $\mathrm{H}$ II regions were 2 times more abundant in the early Milky Way than they are now (cf. Sect. 3.1) and that the number of $\mathrm{H}$ II regions, $n_{\mathrm{H} \mathrm{II}}$, today is about 10000 (cf. Sect. 3.7). Globulette production can take place when the $\mathrm{H}$ II region is still young, $\lesssim 5$ Myrs (cf. Sect. 3.5) and we assume that we can find a similar amount of objects also on the backside of the nebulae (cf. Sect. 3.4). The lifetime of globulettes was estimated to 4 Myrs. The spatial resolution was lower in seven of the $\mathrm{H}$ II regions and according Sect. 3.6, the actual number of globulettes, present in these regions, was estimated to 346 objects.

We list $27 \mathrm{H}$ II regions, where the total area was estimated to $44413 \operatorname{arcmin}^{2}$ and an observed area of $3297 \operatorname{arcmin}^{2}$. The total number of gloublettes was calculated as $G_{T o t}=$ $G_{\mathrm{NOT}}+G_{\mathrm{HST}}=951$ and the average number of globulettes per H II region was estimated to be about 35 . According to our model, the number of globulettes that have formed in the history of the Milky Way (MW) is

$G_{M W}=\frac{T_{\mathrm{II}}}{T_{g l o b}} \cdot \frac{T_{0}}{T_{\mathrm{H} \mathrm{II}}} \cdot n_{\mathrm{H} \mathrm{II}} \cdot f_{B} \cdot f_{\mathrm{H} \mathrm{II}} \cdot \frac{A_{t o t}}{A_{o b s}} \cdot \frac{G_{t o t}}{N_{\mathrm{H} \mathrm{II}}}$.

Using the numerical values from Table 3 we get a total number of globulettes $5.7 \times 10^{10}$ formed in the Milky Way over time, which can be considered a conservative value. It is currently not known what fraction of the globulettes that form FFPs. Their mass and density are likely to affect this fraction but to what extent is currently unknown. Nevertheless, if we assume that $1 \%$ or $10 \%$ of the globulettes form FFPs, then the number of FFPs originating from globulettes is $5.7 \times 10^{8}$ and $5.7 \times 10^{9}$, respectively. 
Fig. 3 Size distribution of the 176 globulettes in 30 Doradus in terms of the mean radii. The distribution peaks at $\sim 5.7 \mathrm{kAU}$. A few larger objects ( $>10 \mathrm{kAU})$ where not included in this study

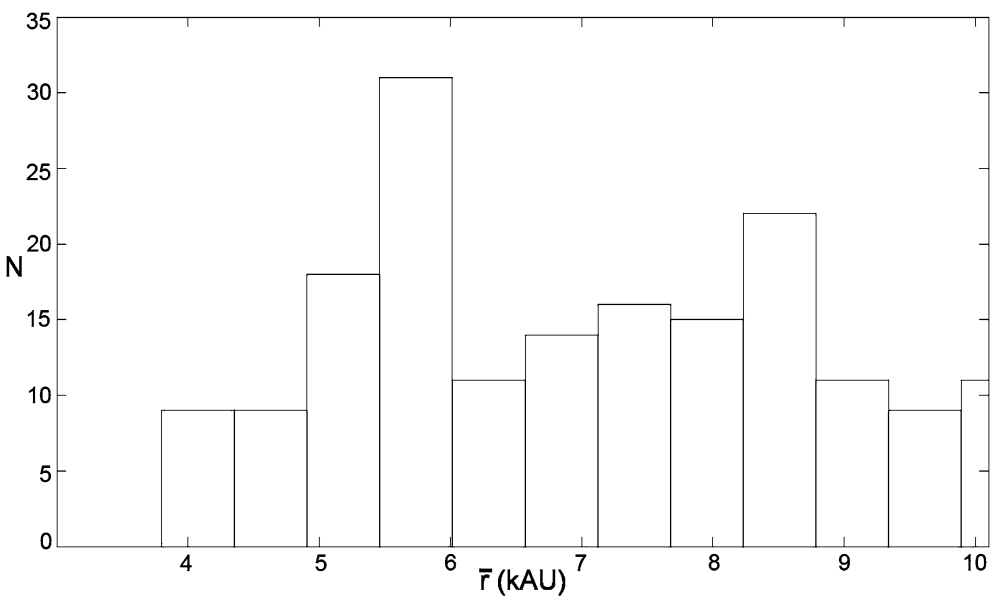

The MOA-2 Galactic bulge microlensing survey (Sumi et al. 2011) found that the Jupiter-sized FFPs are about 1.8 times as common as main-sequence stars. These planets are unbound or bound but very distant $>100 \mathrm{AU}$ from any star. It is unclear if some of these objects are lowmass brown dwarfs or super-Jupiters. The difference between these classes of objects is not well defined (Luhman 2012; Giannini and Lunine 2013). However the population of these objects is expected to arise from a variety of processes, such as in situ formation or from the core accretion theory, see Veras and Raymond (2012) and Sect. 1. However, the number of Jupiter-sized FFPs by Sumi et al. (2011) may be overestimated, due to blending or red noise in the data (Bachelet et al. 2015) or the distribution of the timescale of microlensing events (Di Stefano 2012). Furthermore, Clanton and Gaudi (2017) also found a slightly lower value (1.2-1.4) of FFPs per main sequence star. Recently, Mróz et al. (2017) found, using microlensing, 0.25 per star as an upper limit on the number of Jupiter sized planets.

A more conservative estimate of the number of Jupitersized planets has been calculated by Tutukov and Fedorova (2012) and more recently by Ma et al. (2016), where the FFP population is found to be $\sim 1.8 \cdot 10^{-3}$ of the stellar population, which corresponds to $\sim 10^{8}$ a total number of FFPs. However, these FFPs candidates originate in protoplanetary disks, while the FFPs considered here are formed in situ. Their value is less than the observed value of Sumi et al. (2011) but it is comparable to our result if $1 \%$ of the globulettes form FFPs.

If we increase the factors $f_{\mathrm{H} \text { II }}=2 \rightarrow 3, f_{s}=2 \rightarrow 5$ and $f_{B}=2 \rightarrow 3$, (1) would yield about $2.0 \times 10^{11}$ globulettes. Then by assuming that $10 \%$ of the globulettes form FFPs, the globulettes would then contribute to about $11 \%$ of all FFPs, as estimated by Sumi et al. (2011).

A number of candidates of brown dwarfs and Jupiter mass objects have been found in several nearby, young star clusters and star-forming regions. Drass et al. (2016) have found 160 isolated planetary mass object candidates in the
Orion Nebula. However, we only observe 9 dark objects in this H II region. This can be compared to the Rosette Nebula, where we have 16 times more objects in a smaller area.

We note that the total observed area of the $\mathrm{H}$ II regions is only $7 \%$ of the total nebula area, and in the $27 \mathrm{H} \mathrm{II}$ regions there are 18 nebulae with $<18$ observed globulettes and 8 nebulae with no observed globulettes. In these nebulae, the surveys could simply have missed them or the environment might not be favorable for globulette formation.

In 30 Doradus, which is the closest extragalactic source, many filaments and dust clumps in different sizes have been observed (Indebetouw et al. 2013; Chevance et al. 2016). In this nebula, we discovered 176 globulettes with mean radii less than $10 \mathrm{kAU}$. Their size distribution is shown in Fig. 3 with a peak at about $\sim 5.7 \mathrm{kAU}$. Compared to the globulettes in $\mathrm{H}$ II regions within the Milky Way, these globulettes are rather large. Due to its far distance, the detection limit of the objects in the 30 Doradus is about $4 \mathrm{kAU}$, which means that all smaller globulettes have escaped detection.

\section{Conclusions}

In this paper we have examined $319 \mathrm{H}$ II regions, whereof 28 were observed with a narrowband $\mathrm{H}$-alpha filter. In these $\mathrm{H}$ II regions, we have identified small roundish objects, globulettes, which appear dark in the H-alpha images. We summarize the present work as follows;

1. The total area and observed area of the $27 \mathrm{H} \mathrm{II} \mathrm{regions}$ was estimated to $44413 \operatorname{arcmin}^{2}$ and to $3297 \operatorname{arcmin}^{2}$, respectively.

2. We estimated the mean radii for each globulette found in the H II regions. The majority of objects have radii $<10 \mathrm{kAU}$ but most seem concentrated around $\sim 0.5 \mathrm{kAU}$.

3. Amongst the $27 \mathrm{H}$ II regions, there are 18 nebulae with $<18$ observed globulettes and 8 nebula with no observed globulettes. 
4. In the 30 Doradus, the mean radii had a peak at $\sim 5.7 \mathrm{kAU}$, which was higher than the regions within the Milky Way.

5. The total amount of globulettes was estimated to 778 and by correcting for the factor $f_{s}$ (resolution difference between HST and NOT), the number of globulettes was estimated to 951, which gives an average of 35 globulettes per H II region.

6. Our model gives a conservative estimate on the number of globulettes formed in the history of the Milky Way to about $5.7 \times 10^{10}$. A less conservative estimate gives $2.0 \times 10^{11}$ globulettes.

7. The globulettes could therefore represent a non-negligible source of the FFPs in the Milky Way. For example, if $10 \%$ of the globulettes of our less conservative estimate form FFPs, then the globulettes would contribute to about $11 \%$ of all FFPs, as estimated by Sumi et al. (2011).

Acknowledgements We wish to thank professor Gösta Gahm for many fruitful discussions. We also wish to acknowledge the data provided by the NASA/ESA Hubble Space Telescope (HST), obtained at the Space Telescope Science Institute as well as the Nordic Optical Telescope (NOT).

Open Access This article is distributed under the terms of the Creative Commons Attribution 4.0 International License (http://creative commons.org/licenses/by/4.0/), which permits unrestricted use, distribution, and reproduction in any medium, provided you give appropriate credit to the original author(s) and the source, provide a link to the Creative Commons license, and indicate if changes were made.

\section{References}

Abel, T., Wise, J.H., Bryan, G.L.: Astrophys. J. Lett. 659, L87 (2007) Alvarez, M.A., Bromm, V., Shapiro, P.R.: Astrophys. J. 639, 621 (2006)

Anderson, L.D., Bania, T.M., Balser, D.S., et al.: Astrophys. J. Suppl. Ser. 212, 1 (2014)

Anderson, L.D., Armentrout, W.P., Johnstone, B.M., et al.: Astrophys. J. Suppl. Ser. 221, 26 (2015)

Bachelet, E., Bramich, D.M., Han, C., et al.: Astrophys. J. 812, 136 (2015)

Bally, J., O’Dell, C.R., McCaughrean, M.J.: Astron. J. 119, 2919 (2000)

Bayo, A., Joergens, V., Liu, Y., et al.: Astrophys. J. Lett. 841, L11 (2017)

Beccari, G., Spezzi, L., De Marchi, G., et al.: Astrophys. J. 720, 1108 (2010)

Best, W.M.J., Liu, M.C., Dupuy, T.J., Magnier, E.A.: arXiv:1706. 01883 (2017)

Bica, E., Dutra, C.M., Soares, J., Barbuy, B.: Astron. Astrophys. 404, $223(2003)$

Bihain, G., Rebolo, R., Zapatero Osorio, M.R., et al.: Astron. Astrophys. 506, 1169 (2009)

Bok, B.J., Reilly, E.F.: Astrophys. J. 105, 255 (1947)

Boss, A.P.: Astrophys. J. 694, 107 (2009)

Boucher, A., Lafrenière, D., Gagné, J., et al.: Astrophys. J. 832, 50 (2016)

Bowler, B.P., Liu, M.C., Kraus, A.L., Mann, A.W., Ireland, M.J.: Astrophys. J. 743, 148 (2011)

Brandner, W., Grebel, E.K., Chu, Y.-H., et al.: Astron. J. 119, 292 (2000)
Chabrier, G., Baraffe, I., Allard, F., Hauschildt, P.: Astrophys. J. Lett. 542, L119 (2000)

Chevance, M., Madden, S.C., Lebouteiller, V., et al.: Astron. Astrophys. 590, A36 (2016)

Clanton, C., Gaudi, B.S.: Astrophys. J. 834, 46 (2017)

Crowther, P.A., Conti, P.S.: Mon. Not. R. Astron. Soc. 343, 143 (2003)

Cushing, M.C., Kirkpatrick, J.D., Gelino, C.R., et al.: Astron. J. 147, 113 (2014)

De Marco, O., O’Dell, C.R., Gelfond, P., Rubin, R.H., Glover, S.C.O.: Astron. J. 131, 2580 (2006)

Di Stefano, R.: Astrophys. J. Suppl. Ser. 201, 20 (2012)

Drass, H., Haas, M., Chini, R., et al.: Mon. Not. R. Astron. Soc. 461, 1734 (2016)

Fang, M., Kim, J.S., Pascucci, I., Apai, D., Manara, C.F.: Astrophys. J. Lett. 833, L16 (2016)

Feigelson, E.D., Townsley, L.K., Broos, P.S., et al.: Astrophys. J. Suppl. Ser. 209, 26 (2013)

Foster, T., Brunt, C.M.: Astron. J. 150, 147 (2015)

Freeman, M., Philpott, L.C., Abe, F., et al.: Astrophys. J. 799, 181 (2015)

Gahm, G.F., Grenman, T., Fredriksson, S., Kristen, H.: Astron. J. 133, 1795 (2007)

Gahm, G.F., Persson, C.M., Mäkelä, M.M., Haikala, L.K.: Astron. Astrophys. 555, A57 (2013)

Giannini, E., Lunine, J.I.: Rep. Prog. Phys. 76, 056901 (2013)

Greif, T.H., Johnson, J.L., Bromm, V., Klessen, R.S.: Astrophys. J. 670, 1 (2007)

Grenman, T.: Globulettes-a new class of very small and dense interstellar clouds. Licentiate thesis, Luleå University of Technology (2006)

Grenman, T., Gahm, G.F.: Astron. Astrophys. 565, A107 (2014)

Gum, C.S.: Mem. R. Astron. Soc. 67, 155 (1955)

Haworth, T.J., Facchini, S., Clarke, C.J.: Mon. Not. R. Astron. Soc. 446, 1098 (2015)

Hensberge, H., Pavlovski, K., Verschueren, W.: Astron. Astrophys. 358, 553 (2000)

Herbig, G.H.: Publ. Astron. Soc. Pac. 86, 604 (1974)

Hillenbrand, L.A., Massey, P., Strom, S.E., Merrill, K.M.: Astron. J. 106, 1906 (1993)

Hur, H., Sung, H., Bessell, M.S.: Astron. J. 143, 41 (2012)

Indebetouw, R., Brogan, C., Chen, C.-H.R., et al.: Astrophys. J. 774, 73 (2013)

Jayawardhana, R., Ivanov, V.D.: Science 313, 1279 (2006)

Joergens, V., Bonnefoy, M., Liu, Y., Bayo, A., Wolf, S.: In: 18th Cambridge Workshop on Cool Stars, Stellar Systems, and the Sun, vol. 18, p. 1019 (2015)

Kharchenko, N.V., Piskunov, A.E., Roeser, S., Schilbach, E., Scholz, R.-D.: VizieR Online Data Catalog. vol. 343 (2005)

Kim, K.-T., Koo, B.-C.: Astrophys. J. 596, 362 (2003)

Kitayama, T., Yoshida, N., Susa, H., Umemura, M.: Astrophys. J. 613, 631 (2004)

Kroupa, P., Bouvier, J.: Mon. Not. R. Astron. Soc. 346, 369 (2003)

Kurtz, S., Franco, J.: Rev. Mex. Astron. Astrofís., Ser. Conf. 12, 16 (2002)

Li, Y., Kouwenhoven, M.B.N., Stamatellos, D., Goodwin, S.P.: Astrophys. J. 805, 116 (2015)

Liu, M.C., Magnier, E.A., Deacon, N.R., et al.: Astrophys. J. Lett. 777, L20 (2013)

Lucas, P.W., Roche, P.F.: Mon. Not. R. Astron. Soc. 314, 858 (2000)

Luhman, K.L.: Annu. Rev. Astron. Astrophys. 50, 65 (2012)

Luhman, K.L., Adame, L., D’Alessio, P., et al.: Astrophys. J. Lett. 635, L93 (2005)

Ma, S., Mao, S., Ida, S., Zhu, W., Lin, D.N.C.: Mon. Not. R. Astron. Soc. 461, L107 (2016)

Marasco, A., Debattista, V.P., Fraternali, F., et al.: Mon. Not. R. Astron. Soc. 451, 4223 (2015) 
Mellema, G., Arthur, S.J., Henney, W.J., Iliev, I.T., Shapiro, P.R.: Astrophys. J. 647, 397 (2006)

Menten, K.M., Reid, M.J., Forbrich, J., Brunthaler, A.: Astron. Astrophys. 474, 515 (2007)

Moisés, A.P., Damineli, A., Figuerêdo, E., et al.: Mon. Not. R. Astron. Soc. 411, 705 (2011)

Mookerjea, B., Sandell, G., Jarrett, T.H., McMullin, J.P.: Astron. Astrophys. 507, 1485 (2009)

Moore, B.D., Walter, D.K., Hester, J.J., et al.: Astron. J. 124, 3313 (2002)

Mróz, P., et al.: Nature 548, 183 (2017)

Nakajima, T., Oppenheimer, B.R., Kulkarni, S.R., et al.: Nature 378, 463 (1995)

Noel, B., Joblin, C., Maillard, J.P., Paumard, T.: Astron. Astrophys. 436, 569 (2005)

O’Dell, C.R., Wong, K.: Astron. J. 111, 846 (1996)

O’Dell, C.R., Wen, Z., Hu, X.: Astrophys. J. 410, 696 (1993)

Ohlendorf, H., Preibisch, T., Gaczkowski, B., et al.: Astron. Astrophys. 552, A14 (2013)

Padoan, P., Nordlund, Å.: Astrophys. J. 617, 559 (2004)

Peña Ramírez, K., Béjar, V.J.S., Zapatero Osorio, M.R., Petr-Gotzens, M.G., Martín, E.L.: Astrophys. J. 754, 30 (2012)

Peña Ramírez, K., Béjar, V.J.S., Zapatero Osorio, M.R.: Astron. Astrophys. 586, A157 (2016)

Pietrzyński, G., Graczyk, D., Gieren, W., et al.: Nature 495, 76 (2013)

Quanz, S.P., Goldman, B., Henning, T., et al.: Astrophys. J. 708, 770 (2010)

Rebolo, R., Zapatero Osorio, M.R., Martín, E.L.: Nature 377, 129 (1995)

Reipurth, B., Mikkola, S.: Astron. J. 149, 145 (2015)

Rolleston, W.R.J., Trundle, C., Dufton, P.L.: Astron. Astrophys. 396, 53 (2002)

Román-Zúñiga, C.G., Lada, E.A.: Handbook of Star Forming Regions, Volume I, The Northern Sky, vol. 4, p. 928 (2008)

Sana, H., James, G., Gosset, E.: Mon. Not. R. Astron. Soc. 416, 817 (2011)
Sato, M., Hirota, T., Honma, M., et al.: Publ. Astron. Soc. Jpn. 60, 975 (2008)

Schneider, N., Simon, R., Bontemps, S., Comerón, F., Motte, F.: Astron. Astrophys. 474, 873 (2007)

Schneider, N., Bontemps, S., Motte, F., et al.: Astron. Astrophys. 591, A40 (2016)

Sharpless, S.: Astrophys. J. Suppl. Ser. 4, 257 (1959)

Silk, J.: Astrophys. J. 211, 638 (1977)

Smith, N., Povich, M.S., Whitney, B.A., et al.: Mon. Not. R. Astron. Soc. 406, 952 (2010)

Som, D., Kulkarni, V.P., Meiring, J., et al.: Astrophys. J. 806, 25 (2015)

Stamatellos, D., Whitworth, A.P.: Mon. Not. R. Astron. Soc. 400, 1563 (2009)

Sumi, T., Abe, F., Bond, I.A., et al.: Astrophys. J. 591, 204 (2003)

Sumi, T., Kamiya, K., Bennett, D.P., et al.: Nature 473, 349 (2011)

Thackeray, A.D.: Mon. Not. R. Astron. Soc. 110, 524 (1950)

Tutukov, A.V., Fedorova, A.V.: Astronomy Reports. 56, 305 (2012)

Vargas Álvarez, C.A., Kobulnicky, H.A., Bradley, D.R., et al.: Astron. J. 145, 125 (2013)

Veras, D., Raymond, S.N.: Mon. Not. R. Astron. Soc. 421, L117 (2012)

Wang, L., Kouwenhoven, M.B.N., Zheng, X., Church, R.P., Davies, M.B.: Mon. Not. R. Astron. Soc. 449, 3543 (2015)

Whitworth, A.P., Zinnecker, H.: Astron. Astrophys. 427, 299 (2004)

Wise, J.H., Abel, T.: Astrophys. J. 685, 40-56 (2008)

Wood, D.O.S., Churchwell, E.: Astrophys. J. Suppl. Ser. 69, 831 (1989)

Wyrzykowski, Ł., Rynkiewicz, A.E., Skowron, J., et al.: Astrophys. J. Suppl. Ser. 216, 1 (2015)

Yadav, R.K., Pandey, A.K., Sharma, S., et al.: New Astron. 34, 27 (2015)

Zapatero Osorio, M.R., Béjar, V.J.S., Martín, E.L., et al.: Science 290, $103(2000)$

Zapatero Osorio, M.R., Béjar, V.J.S., Peña Ramírez, K.: Astrophys. J. 842, 1 (2017) 